\title{
Mono-static Synthetic Aperture Radar Interferometry with Arbitrary Flight Trajectories
}

\author{
H. Cagri Yanik and Birsen Yazıcı \\ Electrical, Computer, and System Engineering Department, Rensselaer Polytechnic Institute, \\ Troy, NY 12180 USA
}

\begin{abstract}
We consider a mono-static synthetic aperture radar (SAR) system flying over a scene of interest, making multiple visits. During each visit, antenna is traversing a different arbitrary, but known trajectory. Therefore, the difference in the positions of the antennas, which we refer to as the baseline vector, is arbitrary and changes at each visit. Our objective is to estimate the displacement in the ground topography and reconstruct the scene radiance. We perform a spatio-temporal correlation of the received signals measured by each antenna. This results in a novel model that relates the correlated signal to the displacement. Next, we estimate the displacement in the ground topography and reconstruct the radiance of the scene by using a filtered-backprojection (FBP) type method combined with an entropy minimization technique. Finally, we present numerical experiments to demonstrate the performance of the proposed method.
\end{abstract}

Keywords: Imaging, Interferometry, Synthetic Aperture Radar, Backprojection

\section{INTRODUCTION}

Interferometric synthetic aperture radar (IFSAR) imagery is a technique to determine the topography of a scene. Differential interferometric synthetic aperture radar (DIFSAR) measures the displacement in the topography over time. ${ }^{1}$ Unlike optical and LIDAR imagery, IFSAR works in all-weather conditions, has large area coverage and can monitor an area persistently over time. An IFSAR image is formed by processing the phases of two or more complex SAR images that are obtained with antennas at different heights. Typical IFSAR data is collected by a mono-static SAR system either by traversing a similar trajectory twice (repeat-pass interferometry) or by two tandem antennas (single-pass interferometry). ${ }^{1}$

All of the historic and current space-borne IFSAR systems, including European Remote Sensing Satellite (ERS), Environmental Satellite (Envisat), RADARSAT, Japanese Earth Resources Satellite (JERS) and Advanced Land Observation Satellite (ALOS) are mono-static, repeat-pass systems ${ }^{1-3}$ with the exception of a few systems. ${ }^{4,5}$ Mono-static repeat pass systems have a single mono-static antenna and fly over the same area two or more times with some time apart. An interferogram is formed by using two SAR images that are acquired during each visit. Recently launched Tandem-X system ${ }^{4}$ utilizes two tandem antennas. $\mathrm{In}^{5}$ ground-based antennas collect the signals that are emanated by ERS and an image is formed at each receiver.

In most of the current IFSAR systems, interferograms are formed by working directly with the single look complex (SLC) images. ${ }^{6,7}$ Several algorithms such as range-Doppler, ${ }^{8}$ seismic migration ${ }^{9}$ are used to reconstruct the SLC SAR images. In $^{5}$ a backprojection-based algorithm and in ${ }^{10}$ the chirp scaling algorithm ${ }^{11}$ are used to form SLC images. Current interferometric image formation techniques require both single-pass and repeat-pass antenna systems to traverse the same linear trajectory at different heights.

In this paper, we present a novel differential interferometric synthetic aperture image formation method using a mono-static system traversing arbitrary, different but known trajectories during different visits. We develop a novel model and an inversion method to estimate the displacement in the ground topography and reconstruct the scene radiance. Most of the current IFSAR work in the literature requires SLC images prior to displacement estimation. In our method, the displacement estimation and SAR image formation are performed simultaneously.

Further author information: (Send correspondence to Birsen Yazıcı)

Birsen Yazıcı: E-mail: yazici@ecse.rpi.edu

H. Cagri Yanik: E-mail: yanikh@rpi.edu

Algorithms for Synthetic Aperture Radar Imagery XIX, edited by Edmund G. Zelnio, Frederick D. Garber, Proc. of SPIE Vol. 8394, 83940B · (C) 2012 SPIE · CCC code: 0277-786X/12/\$18 · doi: 10.1117/12.918863 
We assume that the antennas are traversing different, arbitrary, but known trajectories over a scene of interest. Next, we perform a spatio-temporal correlation of the received signals measured by each antenna. This results in a novel model that relates the correlated signal to the displacement in the ground topography and the scene radiance. We next estimate the displacement and reconstruct the scene radiance by using an FBP-type method combined with an entropy minimization technique. Compared to other mono-static IFSAR techniques in the literature our method (1) is computationally efficient, since the displacement estimation is performed while reconstructing the SAR image and (2) accommodates arbitrary flight trajectories.

The rest of the paper is organized as follows: In Section 2, the model for the received signal is given. In Section 3, the forward model for the displacement estimation is derived. Mono-static image formation and the displacement estimation are presented in Section 4. In Section 5, numerical results are provided to demonstrate the performance of our method. Section 6 concludes the paper.

\section{MODEL FOR THE RECEIVED SIGNAL}

We use the following notational conventions throughout the paper. The bold Roman, bold italic and Roman lower-case letters are used to denote variables in $\mathbb{R}^{3}, \mathbb{R}^{2}$ and $\mathbb{R}$, respectively, i.e., $\mathbf{x}=[\boldsymbol{x}, x] \in \mathbb{R}^{3}$, with $\boldsymbol{x} \in \mathbb{R}^{2}$ and $x \in \mathbb{R}$. The calligraphic letters $(\mathcal{F}, \mathcal{K}$ etc. $)$ are used to denote operators.

We assume that there is a mono-static system traversing an arbitrary trajectory over a scene of interest, making multiple visits. Additionally, we assume antenna follows a different, but known trajectory during each visit as depicted in Figure 1. Note that the mono-static system visits the scene with very slow time-scales (typically in the order of hours or days) as compared to the time-scale involved in forming synthetic apertures and imaging.

Let the earth's surface be $\mathbf{x}=[\boldsymbol{x}, \boldsymbol{\psi}(\boldsymbol{x})] \in \mathbb{R}^{3}$, where $\boldsymbol{x} \in \mathbb{R}^{2}$ and $\boldsymbol{\psi}: \mathbb{R}^{2} \rightarrow \mathbb{R}$ is a function for the ground topography. Furthermore, we assume that the scattering takes place in a thin region near the surface. Thus, the reflectivity function at the $k^{\text {th }}$ visit has the form

$$
V(\mathbf{x})=\rho(\boldsymbol{x}) \delta\left(x-\boldsymbol{\psi}^{k}(\boldsymbol{x})\right) ; \quad k=1,2
$$

where $\rho(\boldsymbol{x})$ is the $2 \mathrm{D}$ ground reflectivity function.

We assume that the ground topography undergoes rigid-body displacements from one visit to the next only in the vertical direction. Thus, we define the displacement of a scatterer at point $\mathbf{x}$ between first and second visits, $\zeta_{12}(\boldsymbol{x})$ as

$$
\zeta_{12}(\boldsymbol{x})=\left[0,0, \psi^{2}(\boldsymbol{x})-\psi^{1}(\boldsymbol{x})\right]
$$

Let $s \in\left[0, s_{1}\right]$ denote the slow-time parameter and $\gamma_{k}(s)$ denote the antenna trajectory for the $k^{\text {th }}$ visit, $k=1,2$. The received signal during the $k^{\text {th }}$ visit is given as

$$
f_{k}(s, t)=\int \mathrm{e}^{-\mathrm{i} \omega\left(t-2\left|\gamma_{k}(s)-\mathbf{x}\right| / c_{0}\right)} A_{k}(\omega, \mathbf{x}, s) V(\mathbf{x}) \mathrm{d} \omega \mathrm{d} \mathbf{x}, \quad k=1,2
$$

where $\mathrm{i}=\sqrt{-1}, t$ denotes the fast-time, $c_{0}$ denotes the speed of light in free space, and $A_{k}$ is a slowly varying, complex amplitude function that depends on antenna beam pattern, the transmitted waveform, geometrical spreading factors, etc. ${ }^{12}$

\section{FORWARD MODEL AND THE SPATIO-TEMPORAL CORRELATION OF THE RECEIVED SIGNALS}

In this section we define the spatio-temporal correlation of the received signals and derive the forward model under the incoherent field approximation. ${ }^{13}$ We define the spatio-temporal correlation of $f_{1}$ and $f_{2}$ by

$$
f_{12}\left(s, s^{\prime}, \tau\right)=\int f_{1}(s, \tau) f_{2}^{*}\left(s+s^{\prime}, \tau+t\right) \mathrm{d} t
$$




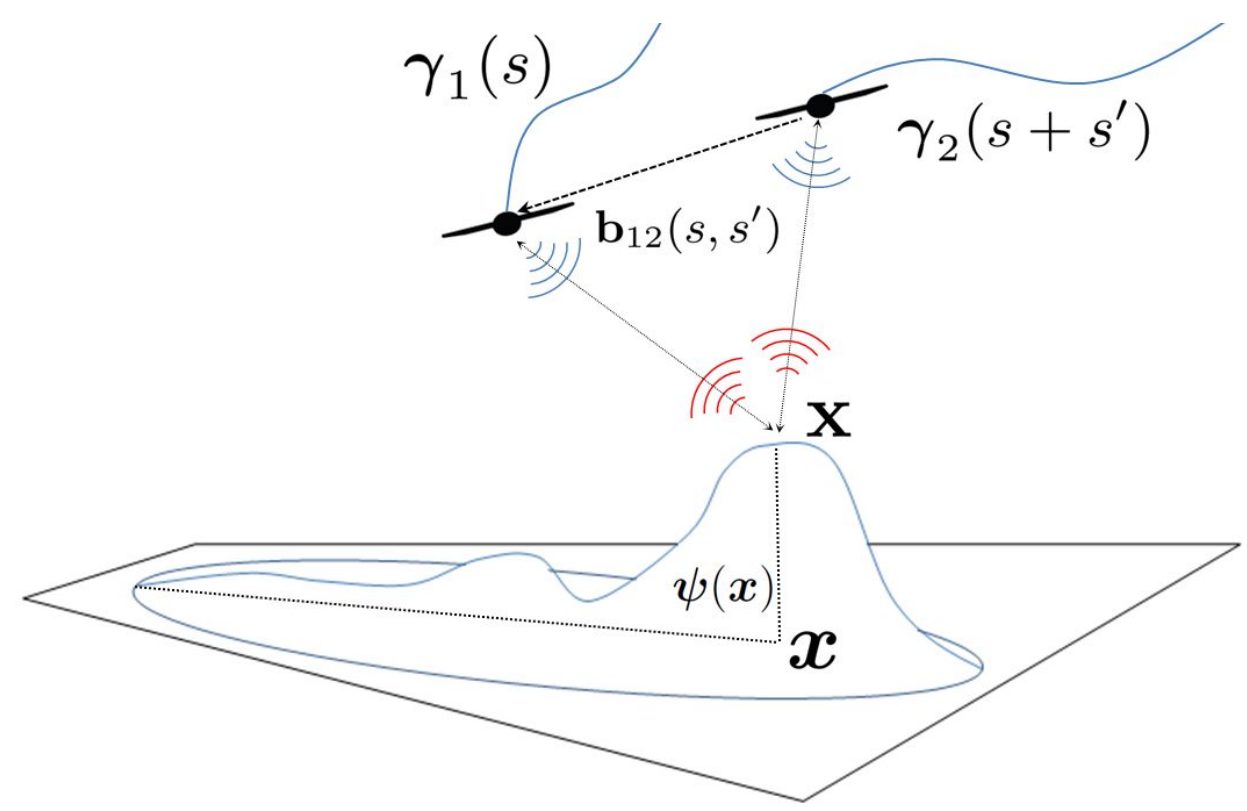

Figure 1. Acquisition geometry for a mono-static system. $\gamma_{1}(s)$ and $\gamma_{2}\left(s+s^{\prime}\right)$ are antenna trajectories during the first and second visits, respectively. $\mathbf{b}_{12}\left(s, s^{\prime}\right)=\gamma_{1}(s)-\gamma_{2}\left(s+s^{\prime}\right)$.

where ${ }^{*}$ denotes complex conjugation and $s$ is the slow-time delay.

Plugging (3) and (2) into (4); making a number of far-field approximations along with the assumption on statistical dependence of the antenna beam patterns under the incoherent-field approximation, ${ }^{13}$ the expected value of the correlated signal becomes

$$
\begin{aligned}
d_{12}\left(s, s^{\prime}, \tau\right) & =E\left[f_{12}\left(s, s^{\prime}, \tau\right)\right] \\
& \approx \mathcal{F}\left[C_{\rho}\right]\left(s, s^{\prime}, \tau\right):=\int \mathrm{e}^{-\mathrm{i} \omega\left(\tau-2 \Delta\left(s, s^{\prime}, \mathbf{x}, \zeta_{12}(\boldsymbol{x})\right) / c_{0}\right)} A_{12}\left(\omega, \mathbf{x}, \zeta_{12}(\boldsymbol{x}), s, s^{\prime}\right) C_{\rho}(\boldsymbol{x}) \mathrm{d} \omega \mathrm{d} \boldsymbol{x}
\end{aligned}
$$

where

$$
\Delta\left(s, s^{\prime}, \mathbf{x}, \zeta_{12}(\boldsymbol{x})\right)=\left(\widehat{\gamma_{1}(s)-\mathbf{x}}\right) \cdot\left(\mathbf{b}_{12}\left(s, s^{\prime}\right)+\zeta_{12}(\boldsymbol{x})\right),
$$

$\mathbf{b}_{12}\left(s, s^{\prime}\right)=\gamma_{1}(s)-\gamma_{2}\left(s+s^{\prime}\right)$ is the baseline vector between the antennas at $\left(s, s+s^{\prime}\right)$ as depicted in Figure 1. $\zeta_{12}(\boldsymbol{x})$ is the displacement of the scatterer at $\boldsymbol{x}$ between first and second visits, defined in (2). $A_{12}$ is a slowly varying function and

$$
C_{\rho}(\boldsymbol{x})=E\left[\rho(\boldsymbol{x}) \rho^{*}(\boldsymbol{x})\right]=E\left[|\rho(\boldsymbol{x})|^{2}\right]
$$

is the scene radiance. ${ }^{13}$ (6) shows that the phase of the correlated signal is the projection of the look direction of the antenna at the first visit onto the sum of the baseline and displacement vectors.

\section{IMAGE FORMATION AND TOPOGRAPHY ESTIMATION}

Our objective is to reconstruct $C_{\rho}(\boldsymbol{x})$ and $\zeta_{12}(\boldsymbol{x})$ from $E\left[f_{12}\left(s, s^{\prime}, \tau\right)\right]$ given in (5). The reconstruction of $C_{\rho}(\boldsymbol{x})$ and $\zeta_{12}(\boldsymbol{x})$ requires the inversion of $\mathcal{F}$. Note that if $\zeta_{12}(\boldsymbol{x})$ is known, an approximate inverse of $\mathcal{F}$ by an FBP operator, $\mathcal{K}$ can be formed such that ${ }^{14,15}$

$$
\tilde{C}_{\rho}(\boldsymbol{x}) \approx C_{\rho}(\boldsymbol{x})=\mathcal{K} \mathcal{F}\left[C_{\rho}\right](\boldsymbol{x})
$$


Since $\zeta_{12}(\boldsymbol{x})$ is unknown, we attempt to reconstruct $C_{\rho}(\boldsymbol{x})$ by an FBP operator, $\mathcal{K}$, for a range of hypothesized $\zeta_{12}(\boldsymbol{x})$ values. We define

$$
\begin{aligned}
\tilde{C}_{\rho}^{\zeta^{\prime}}(\boldsymbol{z}) & \approx \mathcal{K}^{\zeta^{\prime}}\left[d_{12}\right]\left(s, s^{\prime}, \tau\right) \\
& \approx \int \mathrm{e}^{-\mathrm{i} \omega\left[\tau-\Delta\left(s, s^{\prime}, \mathbf{z}, \zeta^{\prime}\right) / c_{0}\right]} d_{12}\left(s, s^{\prime}, \tau\right) B\left(\boldsymbol{z}, \zeta^{\prime}, s, s^{\prime}, \omega\right) \mathrm{d} \omega \mathrm{d} s \mathrm{~d} s^{\prime} \mathrm{d} \tau,
\end{aligned}
$$

for a hypothesized $\zeta^{\prime}$, where $\Delta\left(s, s^{\prime}, \mathbf{z}, \zeta^{\prime}\right)$ is as defined in $(6)$ and $B\left(\boldsymbol{z}, \zeta^{\prime}, s, s^{\prime}, \omega\right)$ is the FBP filter.

If the hypothesized displacement is correct, we expect $\tilde{C}_{\rho}^{\zeta}(\boldsymbol{x})$ to be well-focused. We measure the degree to which the reconstructed image is well-focused by calculating its entropy. Entropy is a well-established figure of merit to measure how well an image is focused. ${ }^{16,17}$ Specifically, we use the Tsallis entropy ${ }^{18}$ which is defined as

$$
E_{r}=\frac{1}{r-1} \sum_{i=1}^{N}\left[p\left(x_{i}\right)-p\left(x_{i}\right)^{r}\right]
$$

where $E_{r}$ is the entropy of the image, $p\left(x_{i}\right)$ is the probability of $x_{i}^{t h}$ pixel value and $r \in \mathbb{R}$. In our experiments, Tsallis entropy is the most accurate and reliable figure of merit among other entropy measures to determine the displacement in the ground topography.

Let $\mathcal{L}=\mathcal{K}^{\zeta} \mathcal{F}$. The kernel $L$ of $\mathcal{L}$ is the point spread function (PSF) of the system which relates the target scene $C_{\rho}(\boldsymbol{x})$ to the reconstructed scene $\tilde{C}_{\rho}(\boldsymbol{x})$. For a perfect reconstruction, $L$ must be a Dirac-delta function. Thus, we choose $\mathcal{K}^{\zeta}$ so that the kernel of $\mathcal{L}$ becomes close to Dirac-delta function whenever the hypothesized ground displacement is equal to its true value.

We use the FBP operator defined in (9) on (5) to form

$$
\begin{aligned}
\tilde{C}_{\rho}^{\zeta^{\prime}}(\boldsymbol{z}) & \approx \mathcal{K} \mathcal{F}\left[C_{\rho}\right]\left(s, s^{\prime}, \tau\right) \\
& \approx \int \mathrm{e}^{\mathrm{i} \omega \Delta\left(s, s^{\prime}, \mathbf{z}, \zeta^{\prime}\right) / c_{0}} B\left(\boldsymbol{z}, \zeta^{\prime}, s, s^{\prime}, \omega\right) D\left(s, s^{\prime}, \omega\right) \mathrm{d} \omega \mathrm{d} s \mathrm{~d} s^{\prime}
\end{aligned}
$$

where $D\left(s, s^{\prime}, \omega\right)=\int d\left(s, s^{\prime}, \tau\right) \mathrm{e}^{\mathrm{i} \omega \tau} \mathrm{d} \tau$.

For some fixed $\boldsymbol{x}=\boldsymbol{z}$ and $\zeta^{\prime}=\zeta$ PSF becomes

$$
L^{\zeta}(\boldsymbol{z}, \boldsymbol{x})=\int \mathrm{e}^{\mathrm{i} \omega\left[\Delta\left(s, s^{\prime}, \mathbf{x}, \zeta\right)-\left.\Delta\left(s, s^{\prime}, \mathbf{z}, \zeta^{\prime}\right)\right|_{\zeta^{\prime}=\zeta}\right] / c_{0}} B\left(\boldsymbol{z}, \zeta, s, s^{\prime}, \omega\right) A_{12}\left(\omega, \mathbf{x}, \zeta, s, s^{\prime}\right) \mathrm{d} \omega \mathrm{d} s .
$$

For $\zeta^{\prime}=\zeta$, since the leading-order contribution to $L^{\zeta^{\prime}}(\boldsymbol{z}, \boldsymbol{x})$ is at $\boldsymbol{x}=\boldsymbol{z},(12)$ can be approximated as

$$
L^{\zeta}(\boldsymbol{z}, \boldsymbol{x}) \approx \int_{\Omega_{\boldsymbol{z}, \zeta}} \mathrm{e}^{\mathrm{i}(\boldsymbol{x}-\boldsymbol{z}) \cdot \boldsymbol{\xi}} B\left(\boldsymbol{z}, \zeta, s^{\prime}, \boldsymbol{\xi}\right) A_{12}\left(\mathbf{x}, \zeta, s^{\prime}, \boldsymbol{\xi}\right) \eta\left(\boldsymbol{z}, \boldsymbol{z}, \boldsymbol{\xi}, s^{\prime}\right) \mathrm{d} \boldsymbol{\xi}
$$

where $\eta\left(\boldsymbol{z}, \boldsymbol{z}, \boldsymbol{\xi}, s^{\prime}\right)$ is the Jacobian coming from the change of variables

$$
(\omega, s) \rightarrow \boldsymbol{\xi}=\frac{\omega}{c_{0}} \boldsymbol{\Xi}\left(s, s^{\prime}, \boldsymbol{z}, \boldsymbol{z}, \zeta\right) \approx-\left.\frac{\omega}{c_{0}} \nabla_{\boldsymbol{x}} \Delta\left(s, s^{\prime}, \boldsymbol{x}, \zeta\right)\right|_{\boldsymbol{x}=\boldsymbol{z}}
$$

Substituting (13) into (8) results in

$$
\tilde{C}_{\rho}^{\zeta}(\boldsymbol{z}) \approx \int_{\Omega_{\boldsymbol{z}, \zeta}} \mathrm{e}^{\mathrm{i}(\boldsymbol{x}-\boldsymbol{z}) \cdot \boldsymbol{\xi}} B\left(\boldsymbol{z}, \zeta, s^{\prime}, \boldsymbol{\xi}\right) A_{12}\left(\mathbf{x}, \zeta, s^{\prime}, \boldsymbol{\xi}\right) \eta\left(\boldsymbol{z}, \boldsymbol{z}, \boldsymbol{\xi}, s^{\prime}\right) C_{\rho}(\boldsymbol{x}) \mathrm{d} \boldsymbol{\xi} \mathrm{d} s^{\prime} \mathrm{d} \boldsymbol{x}
$$

where $\Omega_{\boldsymbol{z}, \zeta}$ is referred to as the data collection manifold at $\boldsymbol{z}$ such that

$$
\Omega_{\boldsymbol{z}, \zeta}=\left\{\boldsymbol{\xi}=\frac{\omega}{c_{0}} \mathbf{\Xi}\left(\boldsymbol{z}, \boldsymbol{z}, s, s^{\prime}, \zeta\right) \mid A_{12}\left(\mathbf{x}, \zeta, s^{\prime}, \boldsymbol{\xi}\right) \neq 0\right\} .
$$


We select the filter for FBP operator as

$$
B\left(\boldsymbol{z}, \zeta, s^{\prime}, \boldsymbol{\xi}\right)=\frac{\chi_{\Omega_{\boldsymbol{z}, \zeta}}}{\eta\left(\boldsymbol{z}, \boldsymbol{z}, \boldsymbol{\xi}, s^{\prime}\right)} \frac{A_{12}^{*}\left(\mathbf{x}, \zeta, s^{\prime}, \boldsymbol{\xi}\right)}{\left|A_{12}\left(\mathbf{x}, \zeta, s^{\prime}, \boldsymbol{\xi}\right)\right|^{2}}
$$

where $\chi_{\Omega_{\boldsymbol{z}, \zeta}}$ is a smooth cut-off function that prevents division by zero and $\frac{1}{\eta\left(\boldsymbol{z}, \boldsymbol{z}, \boldsymbol{\xi}, s^{\prime}\right)}$ is

$$
\frac{1}{\eta\left(\boldsymbol{z}, \boldsymbol{z}, \boldsymbol{\xi}, s^{\prime}\right)}=\left|\frac{\partial \boldsymbol{\xi}}{\partial(\omega, s)}\right|
$$

\section{NUMERICAL EXPERIMENTS}

In our numerical simulations, we considered a scene with a size of $22 \mathrm{~km}-\mathrm{by}-22 \mathrm{~km}$. The center of the scene is located at $[0,0] \mathrm{km}$. We take $A_{T R}(\boldsymbol{x}, \omega, s)=1$, which corresponds to an isotropic antenna radiating a delta-like impulse. Then, using the definition of Dirac-delta function and its homogeneity property, we have

$$
f_{k}(s, t) \approx c_{0} \int \delta\left(c_{0} t-2\left|\boldsymbol{\gamma}_{k}(s)-\mathbf{x}\right|\right) V(\mathbf{x}) \mathrm{d} \boldsymbol{x} .
$$

We used a discrete version of (19) to generate our simulation data. We discretize the scene with $512 \times 512$ pixels, where $[-11,-11] \mathrm{km},[0,0] \mathrm{km}$ and $[11,11] \mathrm{km}$ corresponds to the pixels $[1,512],[256,256]$ and $[512,1]$, respectively. In these experiments, we considered a circular flight trajectory $\gamma_{1}(s)=[22 \cos s, 22 \sin s, 10] \mathrm{km}$ for the first visit of the antenna and a slightly disturbed circular trajectory $\gamma_{2}(s)=[22(\cos s+0.1 \cos s \cos 6 s), 22(\sin s+$ $0.1 \sin s \sin 6 s), 10.2] \mathrm{km}$ uniformly sampled for $s \in[0,2 \pi]$ at 256 points. Thus, we have a known, arbitrary baseline vector for each $s$. (See Figure 2)

In these simulations, displacement estimation and scene radiance reconstruction are performed as follows:

1. Calculate $f_{1}(t, s), f_{2}(t, s)$ for the first and the second visits of the scene with antenna trajectories $\gamma_{1}(s)$ and $\gamma_{2}(s)$ respectively,

2. Calculate spatio-temporal correlation of the received signals, $d_{12}=E\left[f_{1}(t, s) f_{2}^{*}(t, s)\right]$,

3. Backproject correlated signal for each hypothesized displacement $(|\zeta| \leq 20, \zeta \in \mathbb{Z})$ with the filter given in (17) and calculate the entropy of the reconstructed image,

4. For the hypothesized displacements which result in the minimum entropy, perform a local-entropy (13by-13 pixels) measurement for each scatterer and assign the displacement which results in the minimum local-entropy for each scatterer,

5. Return the hypothesized displacements which result in the minimum entropy and reconstruct the final image.

First, we considered a single scatterer at $[2.04,-2.04] \mathrm{km}$ with an elevation of $300 \mathrm{~m}$. The displacement in height from one visit to the next is $1 \mathrm{~m}$. The hypothesized displacement $1 \mathrm{~m}$ at the correct location resulted in minimum entropy and reconstructed image is given in Figure 3.

Secondly, we considered the same scene with 3 scatterers at $[-4.55,4.04] \mathrm{km},[4.04,-4.55] \mathrm{km},[4.04,4.04] \mathrm{km}$ with elevations, $200 \mathrm{~m}, 300 \mathrm{~m}, 250 \mathrm{~m}$ and displacements $1 \mathrm{~m},-1 \mathrm{~m}$ and $2 \mathrm{~m}$ respectively. The hypothesized displacements $1 \mathrm{~m},-1 \mathrm{~m}$ and $2 \mathrm{~m}$ at the correct locations resulted in minimum entropies among others and reconstructed image is given in Figure 4. 


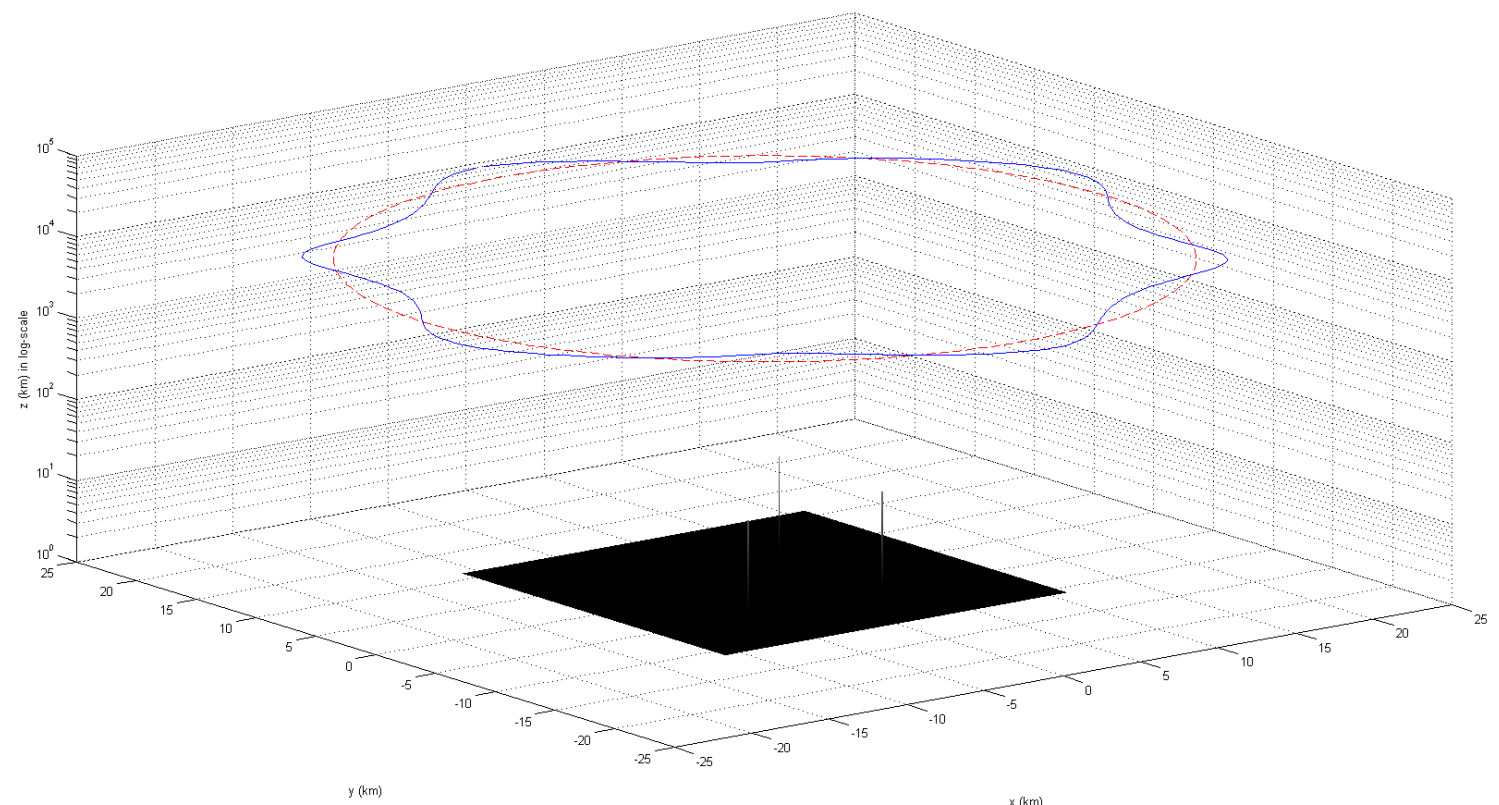

(a)

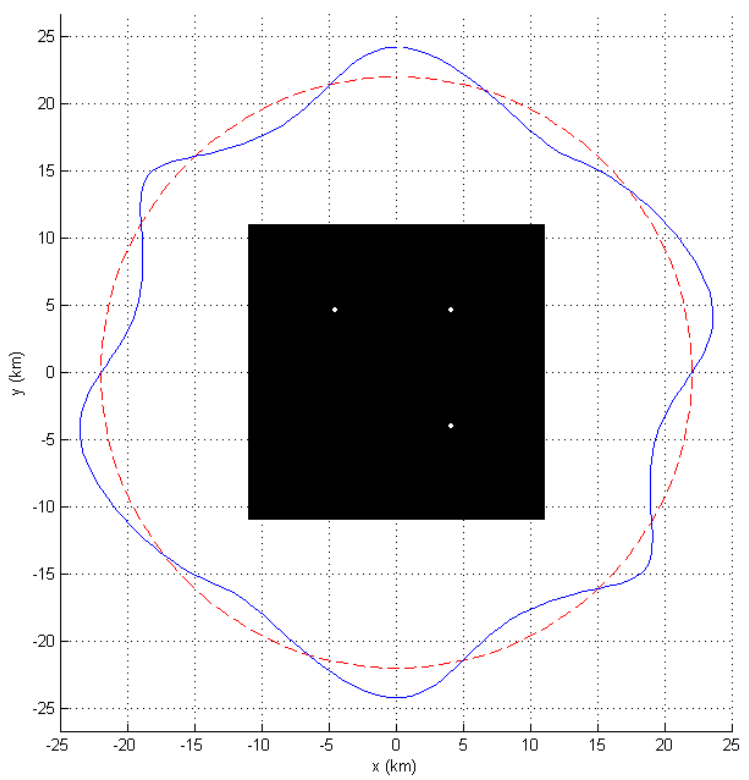

(b)

Figure 2. (a) 3D and (b) 2D views of (dashed line) circular, $\gamma_{1}(s)$ and (solid line) distorted circular flight trajectories, $\gamma_{2}(s)$ for the first and second visits of the antenna, respectively. 


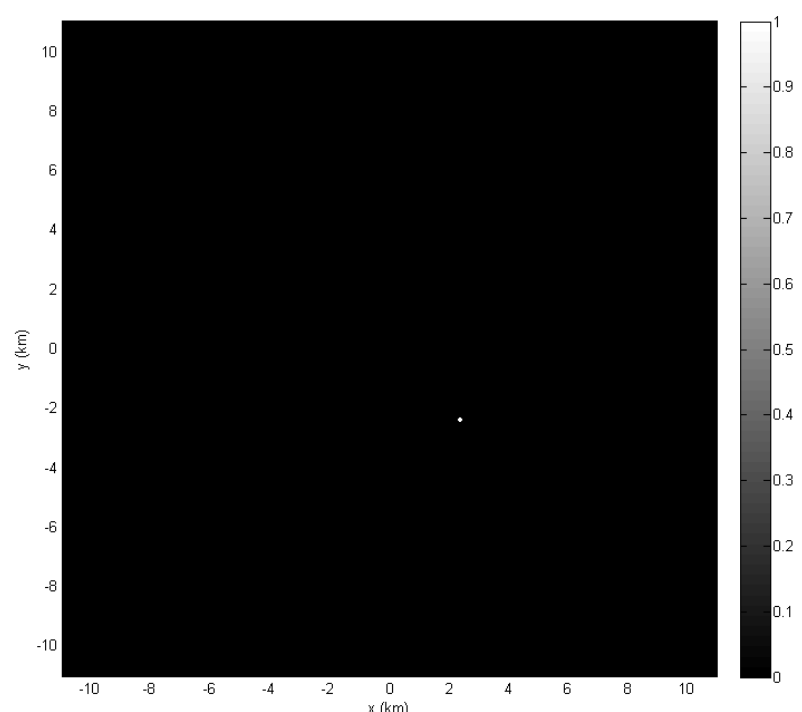

(a)

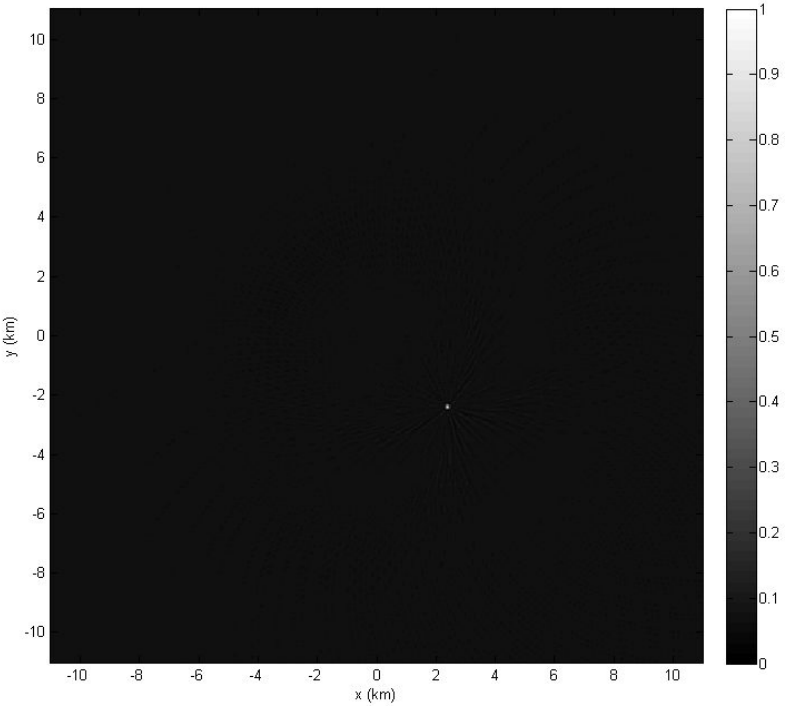

(b)

Figure 3. (a) Original and (b) reconstructed scenes for a single scatterer located at $[2.04,-2.04] \mathrm{km}$ with elevation $200 \mathrm{~m}$ and displacement $1 \mathrm{~m}$. Displacement is estimated correctly.

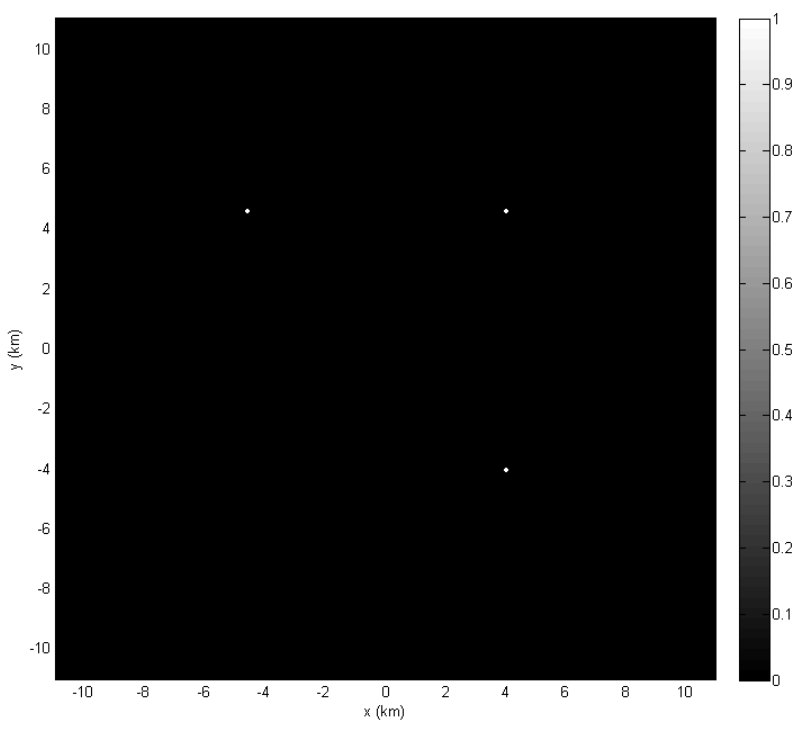

(a)

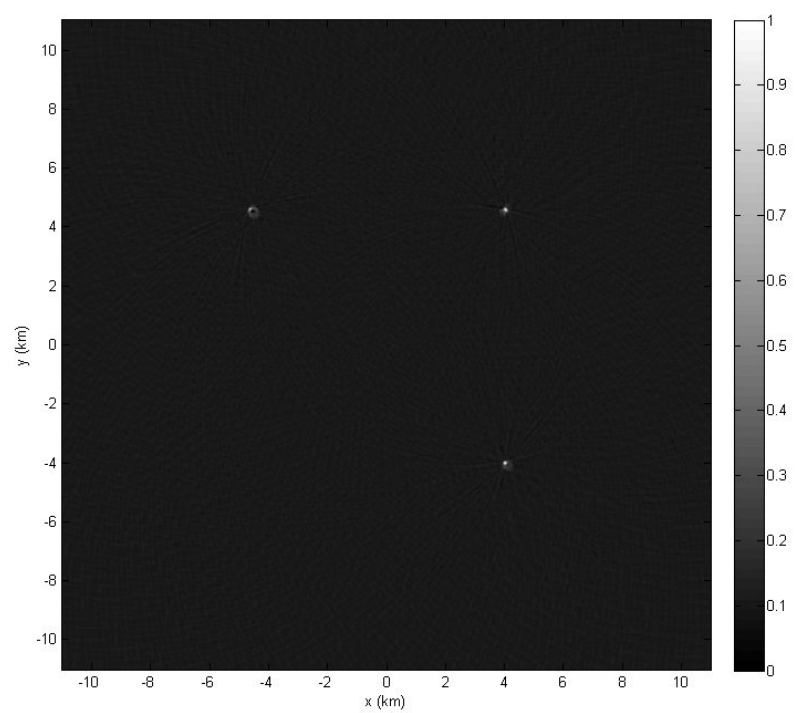

(b)

Figure 4. (a) Original and (b) reconstructed scenes for three scatterers located at $[-4.55,4.04] \mathrm{km},[4.04,-4.55] \mathrm{km}$, $[4.04,4.04] \mathrm{km}$ with elevations, $200 \mathrm{~m}, 300 \mathrm{~m}, 250 \mathrm{~m}$ and displacements $1 \mathrm{~m},-1 \mathrm{~m}$ and $2 \mathrm{~m}$ respectively. Displacements are estimated correctly. 


\section{CONCLUSION}

We presented a novel interferometric synthetic aperture image formation method using a mono-static system traversing arbitrary, different but known trajectories during different visits to estimate the displacement in the ground topography and reconstruct the scene radiance. Compared to other mono-static IFSAR techniques in the literature our method accommodates arbitrary flight trajectories and it is computationally efficient, since the displacement estimation is performed while reconstructing the SAR image and image registration step in the classical IFSAR methods is bypassed. In our future work, we will discuss applying the same technique to multi-static systems.

\section{Acknowledgment}

This work is supported by the Air Force Office of Scientific Research (AFOSR) under the agreements FA9550-071-0363 and FA9550-09-1-0013, by the National Science Foundation (NSF) under Grant No. CCF-08030672 and by the U.S. Dept. of Commerce, National Institute of Standards and Technology (NIST) under 70NANB10H018.

\section{REFERENCES}

[1] Hanssen, R., [Radar interferometry: data interpretation and error analysis], vol. 2, Kluwer Academic Pub (2001).

[2] Rosen, P., Hensley, S., Joughin, I., Li, F., Madsen, S., Rodriguez, E., and Goldstein, R., "Synthetic aperture radar interferometry," Proceedings of the IEEE 88(3), 333-382 (2000).

[3] Cherniakov, M. and Moccia, A., [Bistatic radar: emerging technology], John Wiley \& Sons (2008).

[4] Fritz, T., Rossi, C., Yague-Martinez, N., Rodriguez-Gonzalez, F., Lachaise, M., and Breit, H., "Interferometric processing of tandem-x data," in [IEEE International Geoscience and Remote Sensing Symposium (IGARSS)], 2428-2431, IEEE (2011).

[5] Duque, S., Lopez-Dekker, P., and Mallorqui, J., "Single-pass bistatic sar interferometry using fixed-receiver configurations: Theory and experimental validation," IEEE Transactions on Geoscience and Remote Sensing 48(6), 2740-2749 (2010).

[6] Lombardini, F., "Differential tomography: a new framework for sar interferometry," IEEE Transactions on Geoscience and Remote Sensing 43(1), 37-44 (2005).

[7] Budillon, A., Ferraiuolo, G., Pascazio, V., and Schirinzi, G., "Multichannel sar interferometry via classical and bayesian estimation techniques," EURASIP journal on applied signal processing 2005, 3180-3193 (2005).

[8] Bennett, J. R. and Cumming, I. G., "A digital processor for the production of seasat synthetic aperture radar imagery," in [SURGE Workshop, ESRIN, Frascati, Italy, July 1979, ESA Reprint SP-154],

[9] Prati, C. and Rocca, F., "Limits to the resolution of elevation maps from stereo sar images," International Journal of Remote Sensing 11, 2215-2235 (1990).

[10] Qiu-ling, Z. and Yao-bing, L., "The study on imaging algorithm of formation flying satellites insar system," in [1st Asian and Pacific Conference on SAR], 27-31, IEEE (2007).

[11] Raney, R., Runge, H., Bamler, R., Cumming, I., and Wong, F., "Precision sar processing using chirp scaling," Geoscience and Remote Sensing, IEEE Transactions on 32(4), 786-799 (1994).

[12] Nolan, C. J. and Cheney, M., "Synthetic aperture inversion," Inverse Problems 18, 221-236 (2002).

[13] Barrett, H., Myers, K., and Rathee, S., [Foundations of image science], vol. 44, Wiley-Interscience (2004).

[14] Yarman, C. and Yazıcı, B., "Synthetic aperture hitchhiker imaging," IEEE Transactions on Image Processing 17, 2156-2173 (2008).

[15] Yarman, C., Yazıcı, B., and Cheney, M., "Bistatic synthetic aperture radar imaging for arbitrary flight trajectories," IEEE Transactions on Image Processing 17, 84-93 (2008).

[16] Yegulalp, A., "Minimum entropy sar autofocus," in [Adaptive Sensor Array Processing (ASAP) Workshop, MIT Lincoln Laboratory, Lexington, MA], (1999).

[17] Wang, J. and Liu, X., "Sar minimum-entropy autofocus using an adaptive-order polynomial model," Geoscience and Remote Sensing Letters, IEEE 3(4), 512-516 (2006).

[18] Tsallis, C., "Possible generalization of boltzmann-gibbs statistics," Journal of statistical physics 52(1), 479-487 (1988). 